\title{
ON NONLINEAR DYNAMIC ANALYSIS USING SUBSTRUCTURING AND MODE SUPERPOSITION
}

\author{
KLAUS-JÜRGEN BATHE and SHERYL GRACEWSKI \\ Department of Mechanical Engineering, Massachusetts Institute of Technology, Cambridge, MA 02139, U.S.A.
}

\begin{abstract}
The solution of nonlinear dynamic equilibrium equations using mode superposition and substructuring is studied. The objective is to design schemes that in some analyses can significantly decrease the computational effort involved when compared to a complete direct integration solution. Specific schemes for mode superposition analysis and substructuring are proposed. These techniques have been implemented in ADINA. The results of a few sample analyses are presented and recommendations are given on the use of these procedures in practical analysis.
\end{abstract}

\section{NTRODUCTION}

The analysis of the nonlinear dynamic response of large finite element systems has become of much interest during recent years. The problems to be considered can quite generally be divided into wave propagation problems and structural vibration problems. Both types of problems are solved using an incremental step-by-step solution of the governing equations of motion.

In this paper we consider the solution of structural vibration problems only. These problems can be solved effectively using an implicit time integration scheme with a-modified Newton iteration in each time step. The solution requires that for each of the time steps $\Delta t, 2 \Delta t$, $3 \Delta t, \ldots, n \Delta t$, where $n$ is the total number of time steps considered, the incremental dynamic equilibrium equations be established and then solved using equilibrium iterations. The evaluation of the incremental equilibrium equations involves as a significant computational expense the calculation and the triangular factorization of the effective tangent stiffness matrix, and the equilibrium iterations require the calculation of out-of-balance nodal point force vectors and the forward reduction and backsubstitution of these vectors, until the corrections to the incremental quantities are sufficiently small. The complete solution process is summarized, for example in Table 1 of Refs. $[1,2]$.

Since a large part of the cost in this incremental analysis lies in the calculation of the tangent stiffness matrices and the equilibrium iterations, it is desirable that, without loss of solution stability and accuracy, a new tangent stiffness matrix is only very infrequently formed and equilibrium iterations be only performed when really necessary. Mathematical analysis and practical experience show that because of stability and accuracy considerations, equilibrium iterations are best performed in each time step. However, depending on the problem considered, a new tangent stiffness matrix need not be calculated in each time step, and indeed in many analyses, the original stiffness matrix can be employed throughout the complete response calculation. Also, in many nonlinear analyses, we only deal with local nonlinearities.

The objective in this paper is to consider the nonlinear dynamic analysis of finite element systems for which the original stiffness matrix can be employed throughout the incremental solution, or which only contain local nonlinearities, and demonstrate how the response can be calculated effectively using the basic principles of mode superposition and substructuring.
The possible use of mode superposition in nonlinear analysis is quite natural, because mathematically only a change of basis to a computationally more effective system of equations is performed $[1,3]$. The method of mode superposition has already been applied in nonlinear analysis [4-6], but the effectiveness of the schemes employed in actual practical analyses is still questionable.

The possible use of substructuring on the linear degrees of freedom is equally natural, if we recognize that the solution of the incremental equations involves an "effective stiffness matrix" on which we operate as in static analysis[1-3]. However, there are some important questions with regard to the actual implementation of a substructuring scheme, and these must be addressed in detail in order to arrive at an effective solution strategy.

In the paper we first present the general equations that we employ in an incremental nonlinear dynamic analysis. We then discuss how we use mode superposition and substructuring procedures to reduce the computational effort of solution. The proposed techniques are only effective in the solution of certain nonlinear systems, but then they may yield a significantly more effective solution than the use of direct integration of the complete system of equations. The techniques are implemented in ADINA, and we present the results of some sample analyses using the methods. Finally, we give specific recommendations on the practical usage of the techniques.

\section{INCREMENTAL EQUATIONS OF EQULIBRIUM}

The incremental nodal point equilibrium equations for an assemblage of nonlinear finite elements considered here have been discussed in $[1,3]$. Using an implicit time integration scheme and the modified Newton iteration to establish dynamic equilibrium at time $t+\Delta t$, the governing finite element equations are

$$
\begin{aligned}
\mathbf{M}^{t+\Delta t} \ddot{U}^{(i)}+C^{t+\Delta t} \dot{U}^{(i)}+{ }^{t} \mathbf{K} \Delta \mathbf{U}^{(i)} & \\
& ={ }^{t+\Delta t} \mathbf{R}-{ }^{t+\Delta t} \mathbf{F}^{(i-1)} / i=1,2,3, \ldots 1
\end{aligned}
$$

where

$$
{ }^{t+\Delta t} \mathrm{U}^{(i)}={ }^{1+\Delta t} \mathrm{U}^{(i-1)}+\Delta \mathrm{U}^{(i)}
$$

and $\mathbf{M}=$ constant mass matrix, $\mathbf{C}=$ constant damping matrix, ${ }^{t} \mathbf{K}=$ tangent stiffness matrix at time $t,{ }^{t+\Delta t} \mathbf{R}=$ external nodal point load vector due to body forces, surface loads and concentrated loads, ${ }^{t+\Delta x} \mathbf{F}^{(t-1)}=$ nodal point force vector equivalent to the element stresses that 
correspond to the displacements ${ }^{t+\Delta t} U^{(i-1)},{ }^{t+\Delta t} U^{(i)}=$ vector of nodal point displacements at the end of iteration $(i)$ and time $t+\Delta t$, with derivatives denoted by superposed dots.

For solution of eqn (1), the velocities ${ }^{t+\Delta} \dot{U}^{(t)}$ and accelerations ${ }^{t+\Delta t} \ddot{U}^{(i)}$ are expressed in terms of $\Delta \mathbf{U}^{(i)}$ and known quantities using a time integration scheme as discussed in $[1,3]$. Also, it should be noted that eqn (1) represents the governing equilibrium equations of a very general finite element system with geometric and/or material nonlinearities. The geometric nonlinearities may be due to large displacements and large strains and the material nonlinearities may be due to elastic-plastic, hypo-elastic or other material behavior.

The major computational expense in the solution of eqn (1) lies in the evaluation of ' $K$, its factorization and then the equilibrium iterations. Mathematical analysis and practical experience show that the equilibrium iterations are best always performed, and guidelines for the use of some available procedures to accelerate the convergence of the iterations have been presented in [7]. To reduce the computational effort in the solution of eqn (1) we consider now the use of mode superposition and substructuring.

\subsection{Use of mode superposition}

In principle, the use of mode superposition simply involves a coordinate transformation from the finite element displacement coordinates to the modal coordinates. This change of basis is particularly effective if only a few modal coordinates need be employed. Considering the nonlinear dynamic analysis operating on eqn (1), the change of basis could be performed in each time step using the mode shapes corresponding to time $t$. However, such a procedure would require the solution of the generalized eigenproblem ${ }^{\prime} K^{t} \phi={ }^{\prime} \omega^{2} \mathbf{M}^{t} \phi$, where the superscript " $t$ " indicates that the stiffness matrix, free vibration mode shapes and frequencies correspond to the instantaneous equilibrium configuration at time $t$. It is questionable whether such a scheme would be effective.

When solving eqn (1) it is important to recognize that the new tangent stiffness matrix ' $K$ is only calculated to speed up the convergence of the iteration. In the analysis of some problems it may well be more efficient not to establish a new stiffness matrix in each time step and instead iterate a few more times. In particular, in the analysis of some systems it may be most effective to use the original stiffness matrix corresponding to time 0 throughout the response analysis. In this case, the governing finite element equations for the solution of the response at time $t+\Delta t$ are

$$
\begin{aligned}
\mathbf{M}^{t+\Delta t} \ddot{U}^{(i)}+C^{t+\Delta t} \dot{U}^{(i)}+{ }^{0} \mathbf{K} \underset{{ }^{t+\Delta t}}{\Delta \mathbf{U}^{(i)}} & \\
& ={ }^{t+\Delta t} \mathbf{R}-{ }^{(i-1)} / i=1,2,3, \ldots
\end{aligned}
$$

where ${ }^{0} \mathbf{K}$ is the stiffness matrix corresponding to the configuration and material properties at time 0 . Using eqn (3), a constant effective stiffness matrix is formed in the time integration, and thus only one triangular matrix factorization is needed in the calculation of the dynamic response. All nonlinearities are taken fully into account in the evaluation of the vector ${ }^{t+\Delta t} \mathbf{F}^{(i-1)}$. However, because a constant stiffness matrix is employed, the solution generally requires more equilbrium iterations, and clearly, it is only advantageous to use eqn (3) in the response calculations if the total number of numerical operations is less than in using eqn (1).
The required computations in solving eqn (3) can, in the analysis of some types of problems (which are discussed in Section 3), be reduced significantly using the concepts of mode superposition [4]. The basic step in this solution is a change of basis from the $n$ nodal point displacements to $p$ modal generalized displacements, $p \ll n$, prior to the step-by-step solution. In this transformation we use

$$
{ }^{t+\Delta t} \mathrm{U} \doteq \boldsymbol{\Phi}^{t+\Delta t} \mathbf{X}
$$

where

$$
{ }^{t+\Delta t} X=\left[\begin{array}{r}
t+\Delta t \\
{ }^{t} x_{1} \\
\vdots \\
t+\Delta t \\
{ }^{2} x_{p}
\end{array}\right] ; \Phi=\left[\phi_{1}, \ldots, \phi_{p}\right]
$$

The values ${ }^{t+\Delta r} x_{1}$ are the generalized modal displacements at time $t+\Delta t$ and the vectors $\phi_{i}$ are the eigenvectors of the generalized eigenproblem,

$$
{ }^{0} \mathbf{K} \phi_{i}=\omega_{\mathbf{1}}^{2} \mathbf{M} \phi_{i}
$$

where the $\omega_{i}$ are the natural circular frequencies of the linearized system at time 0 .

The "approximately equal sign" in eqn (4) expresses the fact that an approximation to the solution of eqn (3) is obtained because $p<n$. However, it must be noted that by selecting $p$ sufficiently large, the error in the solution can be made arbitrarily small.

Substituting from eqn (4) into eqn (3) we obtain

$$
\begin{aligned}
{ }^{t+\Delta t} \mathbf{X}^{(i)}+\Lambda^{t+\Delta t} \dot{X}^{(i)}+\Omega^{2} \Delta \mathbf{X}^{(i)} \\
=\Phi^{T}\left({ }^{t+\Delta t} \mathbf{R}-{ }^{t+\Delta t} \mathbf{F}^{(i-t)}\right)
\end{aligned}
$$

where $\Omega^{2}$ is a diagonal matrix listing the circular frequencies squared on its diagonal,

$$
\Omega^{2}=\left[\begin{array}{cc}
\omega_{1}^{2} & \\
\cdot & \cdot \\
& \omega_{p}^{2}
\end{array}\right]
$$

and assuming proportional damping, we can also write,

$$
\Lambda=\left[\begin{array}{cc}
2 \xi_{1} \omega_{1} & \\
& \cdot \\
& 2 \xi_{p} \omega_{p}
\end{array}\right]
$$

where the $\xi_{i}$ are the modal damping ratios corresponding to the $\omega_{i}$.

It should be noted that, different from linear analysis, the incremental equilibrium equations in the new basis, eqn (7) are still coupled, because the nodal point vector ${ }^{t+\Delta t} F^{(i-1)}$ can only be evaluated once all displacements are known,

$$
{ }^{t+\Delta t} U^{(i-1)}=\sum_{k=1}^{n} \phi_{k}{ }^{t+\Delta t} x_{k}^{(i-1)} .
$$

Therefore, the solution of the $p$ equations in eqn (7) must be performed simultaneously. However, since $\Lambda$ and $\mathbf{\Omega}^{2}$ are diagonal matrices and only $p$ equations are considered, $p \ll n$, the solution of the eigenproblem in eqn (6) plus the step-by-step solution of eqn (7) (using, for example, the Newmark method) can be significantly more cost-effective than the direct step-by-step solution of eqn (3). 


\subsection{Use of substructuring}

Substructuring for solution of eqn (1) can effectively be used in the analysis of large problems with small isolated areas of nonlinearities. In such case we can statically condense the linear degrees of freedom prior to the solution of the incremental equations of equilibrium. In the following, we use the Newmark method of time integration; however, the solution procedure can equally be employed with other direct integration schemes (in ADINA we have implemented the procedure for the Newmark and Wilson integration methods).

Using the Newmark method of time integration, eqn (1) becomes [2,3],

$$
\begin{aligned}
{ }^{t} \hat{\mathbf{K}} \Delta \mathbf{U}^{(i)}= & { }^{t+\Delta t} \mathbf{R}-{ }^{t+\Delta t} \mathbf{F}^{(i-1)} \\
& -\mathbf{M}\left\{a_{0} \mathbf{U}^{(i-1)}-a_{2}{ }^{t} \dot{\mathbf{U}}-a_{3}{ }^{i} \dot{\mathbf{U}}\right\} \\
& -\mathbf{C}\left\{a_{1} \mathbf{U}^{(i-1)}-a_{4}{ }^{\prime} \dot{\mathbf{U}}-a_{5}{ }^{t} \mathbf{U}\right\}
\end{aligned}
$$

where the time integration constants $a_{0} \ldots, a_{5}$ are defined in Appendix A, and

$$
\begin{gathered}
' \hat{\mathbf{K}}={ }^{t} \mathbf{K}+a_{0} \mathbf{M}+a_{1} \mathbf{C} \\
\mathbf{U}^{(i-1)}={ }^{t+\Delta t} \mathbf{U}^{(1-3)}-{ }^{t} \mathbf{U} .
\end{gathered}
$$

Since we now consider the solution of problems for which isolated nonlinearities are present, we can write ' $\hat{\mathbf{K}}$ in the form

$$
{ }^{\prime} \hat{\mathbf{K}}=\hat{\mathbf{K}}+{ }^{\prime} \mathbf{K}^{(\boldsymbol{n} l)}
$$

where $\hat{\mathbf{K}}$ contains all constant elements of ' $\hat{\mathbf{K}}$ (including the mass and damping terms) and ${ }^{~} \mathbf{K}^{(n t)}$ corresponds to all nonlinear (time-dependent) entries in ' $\hat{\mathbf{K}}$. Figure 1 shows schematically the structure of the matrices in eqn (14).

Considering eqn (11) it follows that static condensation or substructuring can be employed on all those degrees of freedom that pertain to constant elements in ' $\hat{\mathbf{K}}$. This means, in essence, that the $\mathbf{L} \mathbf{D ~ L}^{t}$ factorization of ' $\hat{\mathbf{K}}$ on the "linear degrees of freedom" need be performed only once prior to the step-by-step solution, at time 0 , and only the stiffness values of the "nonlinear degrees of freedom" need be updated and factorized at the beginning of each step. However, if the effective load vector is evaluated as shown in eqn (11), the complete vector has to be formed and reduced in each iteration. The efficiency of the equilibrium iterations is greatly improved by rewriting eqn (11) in the form,

$$
{ }^{i} \hat{\mathbf{K}} \Delta \mathbf{U}^{(i)}={ }^{t+\Delta t} \mathbf{R}-{ }^{t+\Delta t} \hat{\mathbf{F}}^{(i-1)}+\mathbf{F}^{\mathrm{M}}+{ }^{t} \mathbf{F}^{C}
$$

where

$$
\begin{aligned}
{ }^{t+\Delta t} \hat{\mathbf{F}}^{(i-1)}={ }^{t+\Delta t} \mathbf{F}^{(i-1)}+\left(a_{0} \mathbf{M}+a_{1} \mathbf{C}\right)^{t+\Delta t} \mathrm{U}^{(i-1)} \\
{ }^{t} \mathbf{F}^{M}=\mathbf{M}\left(a_{0}{ }^{t} \mathbf{U}+a_{2}{ }^{t} \dot{\mathrm{U}}+a_{3}{ }^{t} \dot{\mathrm{U}}\right) \\
\mathrm{F}^{C}=\mathrm{C}\left(a_{1}{ }^{t} \mathbf{U}+a_{4}{ }^{t} \dot{\mathrm{U}}+a_{5}{ }^{t} \dot{\mathrm{U}}\right)
\end{aligned}
$$

Equation (15) is in more detail

$$
\begin{aligned}
& \left\{\left[\begin{array}{ll}
\hat{\mathbf{K}}_{c c} & \hat{\mathbf{K}}_{c a} \\
\hat{\mathbf{K}}_{a c} & \hat{\mathbf{K}}_{a a}
\end{array}\right]+\left[\begin{array}{cc}
0 & 0 \\
0 & \mathbf{K}^{(n l)}
\end{array}\right]\right\}\left[\begin{array}{l}
\Delta \mathbf{U}_{c}^{(i)} \\
\Delta \mathbf{U}_{a}^{(i)}
\end{array}\right]=\left[\begin{array}{l}
t+\Delta t \\
\mathbf{R}_{c} \\
t+\Delta t \\
\mathbf{R}_{a}
\end{array}\right]
\end{aligned}
$$

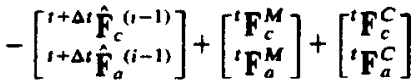

where the $\mathbf{U}_{c}$ degrees of freedom are to be condensed out. Performing the static condensation in the usual way we obtain

$$
\begin{aligned}
& {\left[\left(\hat{\mathbf{K}}_{a a}-\hat{\mathbf{K}}_{a c} \hat{\mathbf{K}}_{c c}^{-1} \hat{\mathbf{K}}_{c a}\right)+{ }^{i} \mathbf{K}^{(n)}\right] \Delta \mathbf{U}_{a}^{(i)}} \\
& =\left({ }^{i}+\Delta t R_{a}+{ }^{t} \mathbf{F}_{a}^{M}+{ }^{\prime} \mathbf{F}_{a}^{C}\right) \\
& -\hat{\mathbf{K}}_{a c} \hat{\mathbf{K}}_{c c}^{-1}\left({ }^{t+\Delta t} \mathbf{R}_{c}+{ }^{t} \mathbf{F}_{c}^{M}+{ }^{t} \mathbf{F}_{c}^{C}\right) \\
& \text { TERM } 1 \\
& \underbrace{-\left({ }^{i+\Delta t} \hat{\mathbf{F}}_{u}^{(i-1)}-\hat{\mathbf{K}}_{\mathrm{ac}} \hat{\mathbf{K}}_{\mathrm{cc}}^{-1 t+\Delta t} \hat{\mathbf{F}}_{c}^{(i-1)}\right)} \\
& \text { TERM } 2
\end{aligned}
$$

Considering eqn (20) it is clear that the expression labelled TERM 1 need only be evaluated once at the beginning of the iteration, because it is independent of the iteration counter $(i)$. In order to identify how the expression labelled TERM 2 can be evaluated effectively, we use the following relation

$$
\begin{array}{r}
{\left[\begin{array}{l}
t+\Delta t \hat{\mathbf{F}}_{c}^{(i-1)} \\
t+\Delta t \hat{\mathbf{F}}_{a}^{(t-1)}
\end{array}\right]=\left[\begin{array}{ll}
\hat{\mathbf{K}}_{c c} & \hat{\mathbf{K}}_{c a} \\
\hat{\mathbf{K}}_{a c} & \hat{\mathbf{K}}_{a a}
\end{array}\right]\left[\begin{array}{l}
t+\Delta t \mathbf{U}_{c}^{(i-1)} \\
{ }^{t+\Delta t} \mathbf{U}_{a}^{(i-1)}
\end{array}\right]} \\
+\left[\begin{array}{c}
0 \\
t+\Delta t \mathbf{F}^{(n) \times i-1)}
\end{array}\right]
\end{array}
$$

where ${ }^{t+\Delta t} F^{(n /)(1-1)}$ represents the nodal point forces corresponding to the element stresses in the nonlinear elements [1.3]. Using eqn (21) we obtain

$$
\begin{aligned}
& { }^{t+\Delta t} \hat{\mathbf{F}}_{a}^{(i-1)}-\hat{\mathbf{K}}_{a \mathbf{K}} \hat{\mathbf{K}}_{\mathrm{c}}^{-1+\Delta t} \hat{\mathbf{F}}_{c}^{(t-1)} \\
& \quad=\left(\hat{\mathbf{K}}_{a a}-\hat{\mathbf{K}}_{a c} \hat{\mathbf{K}}_{c c}^{-1} \hat{\mathbf{K}}_{c a}\right)^{t+\Delta t} \mathrm{U}_{a}^{(i-1)}+{ }^{t+\Delta t} \mathbf{F}^{(n /)(i-1)} .
\end{aligned}
$$

Hence, the static condensation of the $\mathbf{U}_{c}$ displacements

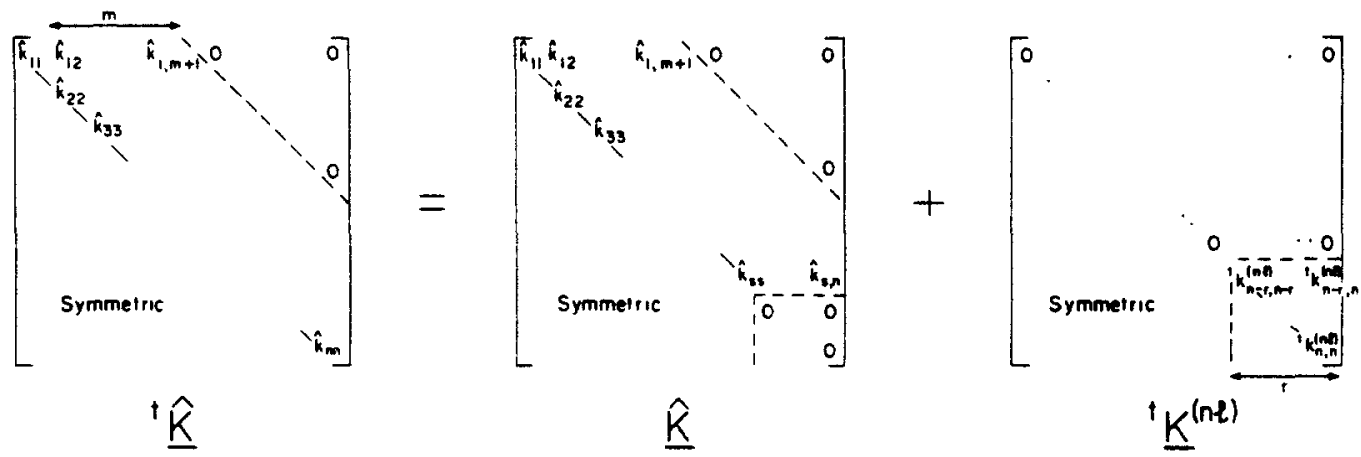

Fig. 1. Schematic of stiffness matrix used in dynamic substructuring. 
yields

$$
\begin{aligned}
\left(\hat{\mathbf{K}}_{r}+{ }^{t} \mathbf{K}^{(\boldsymbol{n})}\right) \Delta \mathbf{U}_{a}^{(i)}={ }^{t+\Delta t} \hat{\mathbf{R}}_{r}-\hat{\mathbf{K}}_{r}{ }^{t+\Delta t} \mathbf{U}_{a}^{(i-1)} & -{ }^{t+\Delta t} \mathbf{F}^{(n) /(i-1)}
\end{aligned}
$$

where

$$
\begin{gathered}
\hat{\mathbf{K}}_{r}=\hat{\mathbf{K}}_{a a}-\hat{\mathbf{K}}_{a c} \hat{\mathbf{K}}_{c c}^{-1} \hat{\mathbf{K}}_{c a} \\
{ }^{t+\Delta t} \hat{\mathbf{R}}_{r}={ }^{t+\Delta t} \mathbf{R}_{a}+{ }^{t} \mathbf{F}_{a}^{M}+{ }^{\prime} \mathbf{F}_{a}^{C}-\hat{\mathbf{K}}_{a c} \hat{\mathbf{K}}_{c c}^{-1} \\
\times\left({ }^{t+\Delta t} \mathbf{R}_{c}+{ }^{t} \mathbf{F}_{c}^{M}+{ }^{\prime} \mathbf{F}_{c}^{C}\right) .
\end{gathered}
$$

It is important to note that in the solution of eqn (23) the vector ${ }^{t+\Delta} \hat{\mathbf{R}} \hat{\mathbf{R}}_{r}$ need only be calculated once at the beginning of the iteration and the iteration is performed only with the $\mathrm{U}_{a}$ degrees of freedom. Once the iteration has converged to the displacements ${ }^{t+\Delta t} U_{a}$, we obtain the displacements ${ }^{i t \Delta t} U_{c}$ using eqn (19),

$$
\hat{\mathbf{K}}_{c c}{ }^{t+\Delta t} \mathbf{U}_{c}={ }^{t+\Delta t} \mathbf{R}_{c}-\hat{\mathbf{K}}_{c a}{ }^{i+\Delta t} \mathbf{U}_{a}+{ }^{t} \mathbf{F}_{c}^{M}+{ }^{t} \mathbf{F}_{c}^{C} .
$$

This solution only requires a forward reduction and backsubstitution because the factorization of $\hat{\mathbf{K}}_{c c}$ was already performed in the static condensation to obtain $\hat{\mathbf{K}}_{\text {r }}$. With the nodal-point displacements known, the corresponding velocities and accelerations can directly be evaluated as given in Appendix A.

The above static condensation procedure is implemented effectively using substructuring. Equations (23)-(25) show that the substructuring can be performed much in the same way as in static analysis, but using the effective stiffness matrix and calculating an effective load vector ${ }^{r+\Delta t} \hat{\mathbf{R}}_{r}$ at the beginning of each time step. Also, since the evaluation of the load vector ${ }^{1+\Delta \prime} \hat{\mathbf{R}}$, involves all displacements, velocities and accelerations corresponding to time $t$, it is necessary to calculate the condensed nodal point displacements (using eqn 26) always immediately after convergence of the iteration for the retained degrees of freedom. On the other hand, in static analysis the condensed nodal-point displacements corresponding to all load levels can be calculated after the complete solution of the retained degrees of freedom has been obtained.

\section{USE OF MODE SUPERPOSITION AND SUESTRUCTURING}

The mode superposition and substructuring procedures described in Sections 2.1 and 2.2 are available in the ADINA computer program. The sample problems described in the following only demonstrate the applicability of the solution procedures. The efficiencies of the methods are discussed in Section 3.2.

\subsection{Sample problems}

The first two sample problems consist of linear structures which have contact regions that are "small" in comparison to the total problem size. In the first example, there are twelve degrees of freedom and the ratio of nodes in contact to total structure nodes is $1: 4$. In the second example, the ratio is increased to 1:16 with a total of 64 degrees of freedom. These two problems are of the type for which the use of mode superposition and substructuring can be effective.

In the third sample problem, the earthquake response of a tall structure is examined. Since the entire structure is allowed to become plastic, dynamic structuring cannot be used. Mode superposition analyses using 1, 4 and 8 modes were compared to the direct integration solution.

All sample problems were solved using the computer program ADINA. For all direct integration solutions, the Newmark method was used.

3.1.1 Control rod drive housing with stops. The 69 product line Control Rod Drive (CRD) housing shown in Fig. 2 is subjected to a sinusoidal ground acceleration with $5 \mathrm{~Hz}$ frequency. The finite element model consists of four beam elements of length, $L$, with a concentrated mass at each nodal point. Values for all constants are given in Fig. 2. Mass proportional loading was used to simulate the ground acceleration since this option is the one available in ADINA.

Two stops were placed at a distance $2 d=0.2 \mathrm{in}$. apart to limit the tip deflection of the CRD housing. Nonlinear elastic springs were used to simulate the contact.

The following three methods of solution were compared: (1) direct integration without substructuring, (2) direct integration with the entire CRD housing as a substructure and the nonlinear springs as the master structure, and (3) mode superposition using 2 modes. A time increment of $0.001 \mathrm{sec}$ and BFGS interations were used for the direct integration solutions. The modified Newton iteration was used for the mode superposition solution, for which a smaller timestep, $\Delta t=0.00035 \mathrm{sec}$ was needed for convergence.

A plot of the tip deflection versus time is shown in Fig. 3. The solid line is the solution from Ref. [8] in which a time increment of $0.0001 \mathrm{sec}$ was used.

3.1.2 Piping example. A 1920 in. long pipe cantilevered at one end and supported at its midpoint and other end is modeled using 32 beam elements as shown in Fig. 4. The supports were modeled with nonlinear elastic-plastic springs with constants also given in the figure. The pipe was loaded by a sinusoidal mass proportional loading of $3 \mathrm{~Hz}$.

Three analyses were carried out: (1) direct integration, no substructures, (2) direct integration with the pipe as a

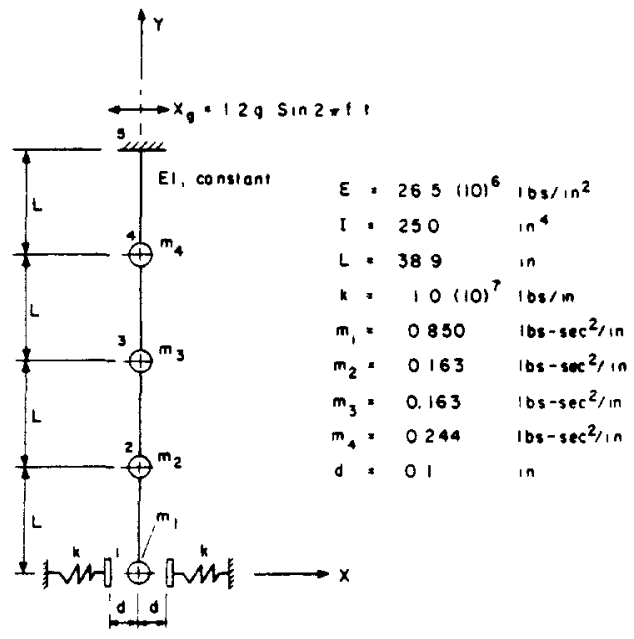

$$
\begin{aligned}
& \text { Q ground accelectotion of } \\
& \qquad \begin{array}{l}
120 \sin 2 \pi 14 \\
x_{9}=386 \mathrm{in} / \mathrm{sec}^{2}
\end{array}
\end{aligned}
$$

Fig. 2. CRD housing with lower support. 
TIME ISECONOS

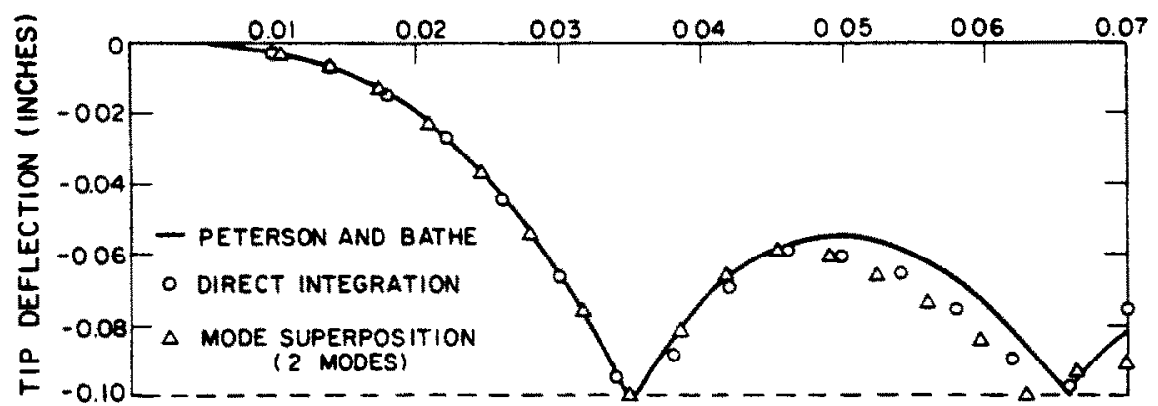

Fig. 3. CRD housing tip deflection.
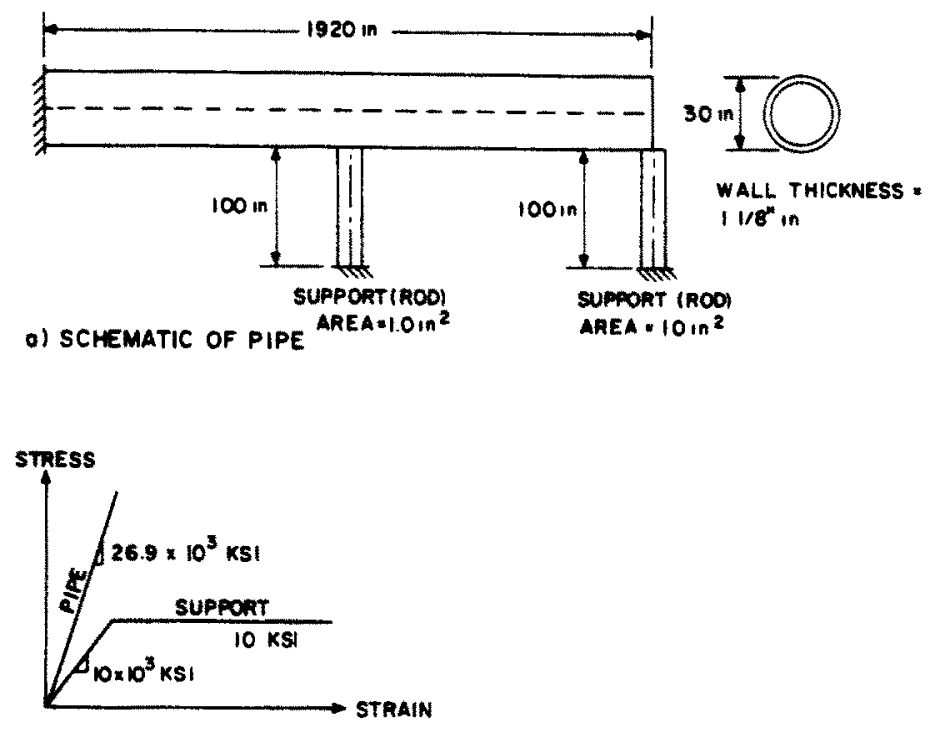

b) STRESS-STRAIN RELATIONS

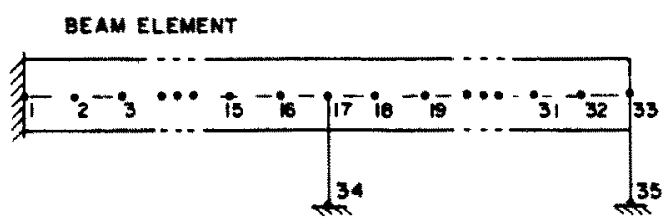

$440 \mathrm{posec} 2$ in LUMPED MASSES AT NOOES 2 TO 32

$224 \mathrm{tbes} 2 \mathrm{e} / \mathrm{in}$ LUMPED MASS AT NOOE 33

c) FINITE ELEMENT MODEL

Fig. 4. Model of pipe with two elastic-plastic supports.

substructure and the two springs as the master structure and (3) mode superposition with 10 and 20 modes. All solutions were performed using equilibrium iterations and a time step of $0.004 \mathrm{sec}$.

Figure 5 shows a plot of the predicted midpoint displacement of the pipe as a function of time. As expected. the solution using substructuring gave the same results as the direct integration solution without substructuring (within the convergence tolerances of the iterations). The mode superposition solution approximates the peak displacement within an error of $10 \%$ when using 10 modes and is much more accurate with 20 modes.

3.1.3 Earthquake response of a tall building. A 34 story shear building was subjected to the North-South Component of the 1940 El Centro Earthquake times two. The finite element model consisted of 34 elastic-plastic truss elements whose masses static yield forces, and stiffness are given in Fig. 6. A similar building was analyzed by Lukkunaprasit $\boldsymbol{e t}$ al. [9].

The solution algorithms used were direct time integration and mode superposition with 1,4 and 8 modes. In all cases the time increment, $\Delta t$, was $0.02 \mathrm{sec}$. The solution was carried out for the first four seconds of the earthquake.

Figures 7-9 show predicted displacement responses in the solution, and Fig. 10 gives a displacement factor at 


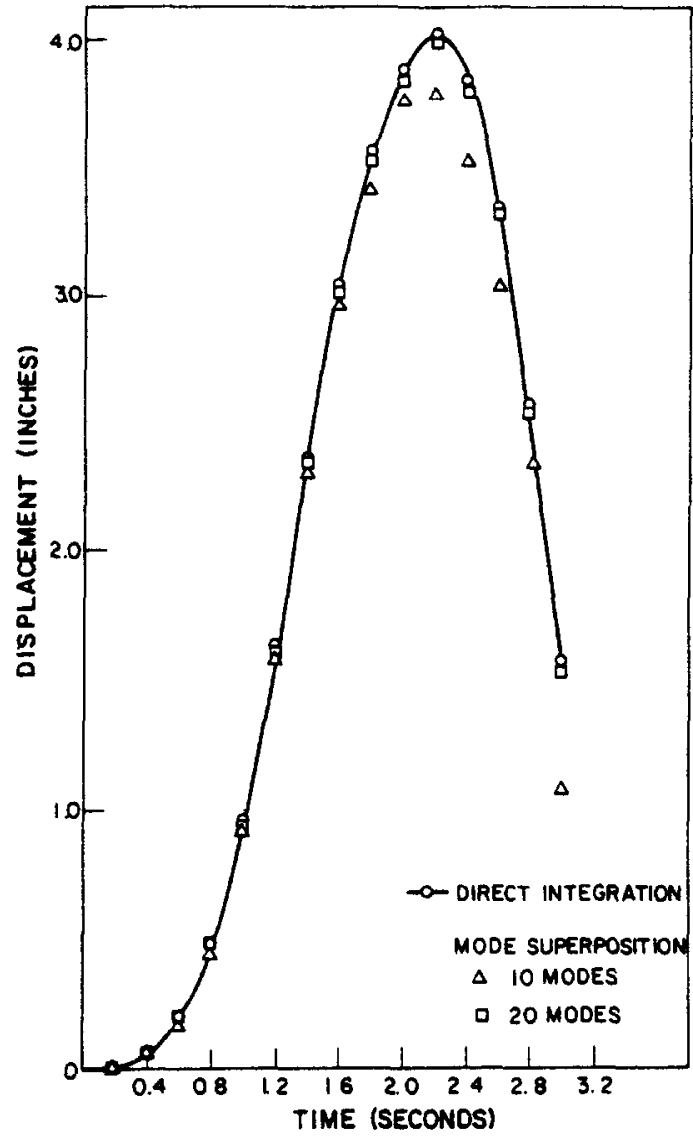

Fig. 5. Predicted midpoint displacement of pipe with two elasticplastic supports.

$t=2.8$ and $4 \mathrm{sec}$ (this factor is defined as strain/(strain at initial yield point)). The response curves show that as the number of modes used is increased the response predicted in the direct integration is approached. Also, as expected, the displacements can be predicted accurately using less modes than are needed for an accurate prediction of the displacement factor, because this factor is given by the derivatives of the displacements.

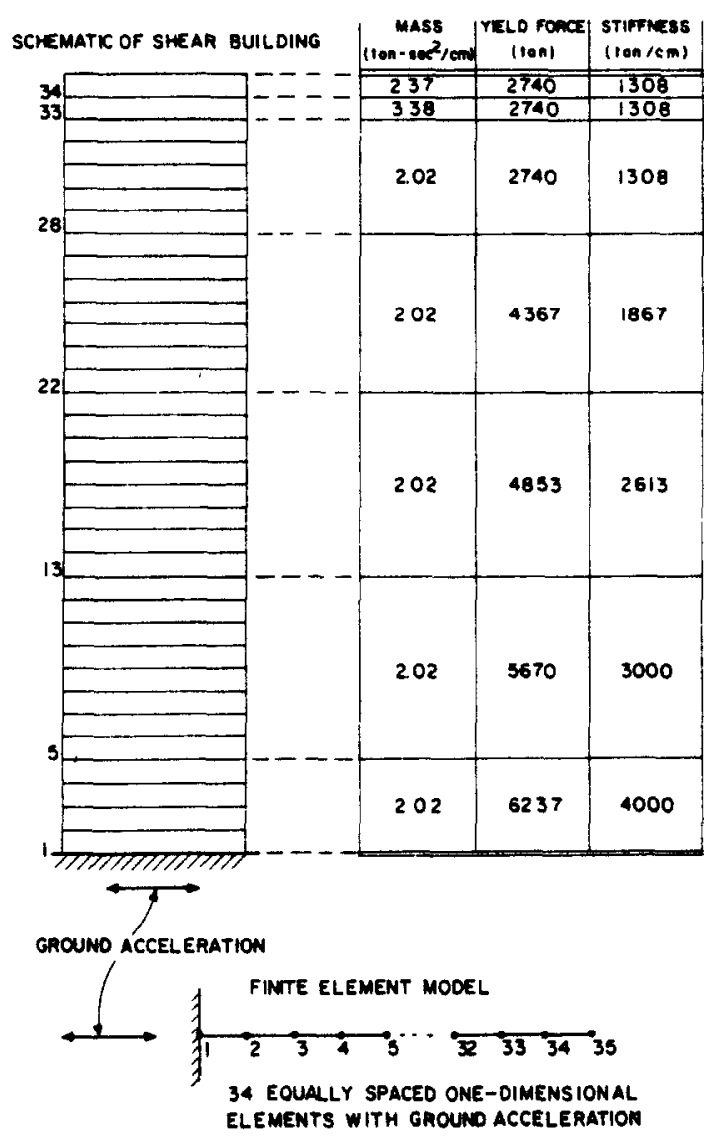

Fig. 6. Model of 34-story shear building.

\subsection{Efficiencies of methods}

In practical analysis it is important to be able to decide in a rational manner when to use the above mode superposition and substructuring procedures. For this reason we want to give some specific guidelines on the usage of the methods.

3.2.1 Mode superposition. The basic premise of the mode superposition procedure is that the transformation

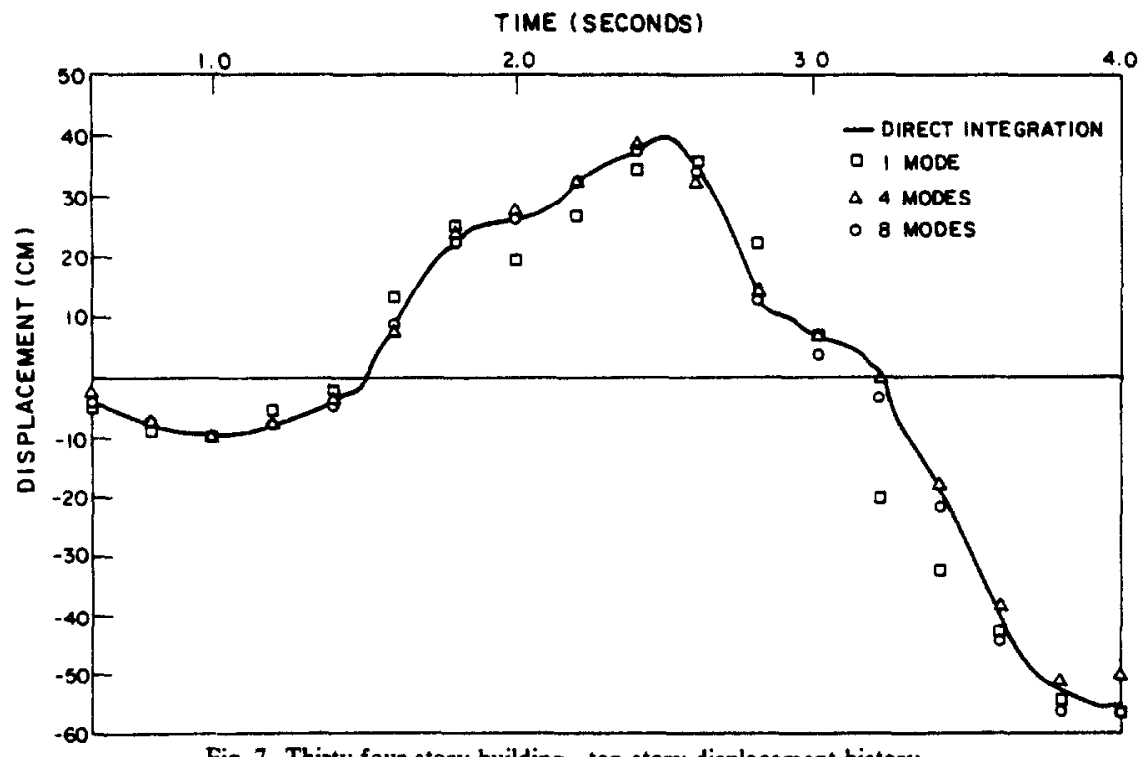

Fig. 7. Thirty-four story building-top story displacement history. 


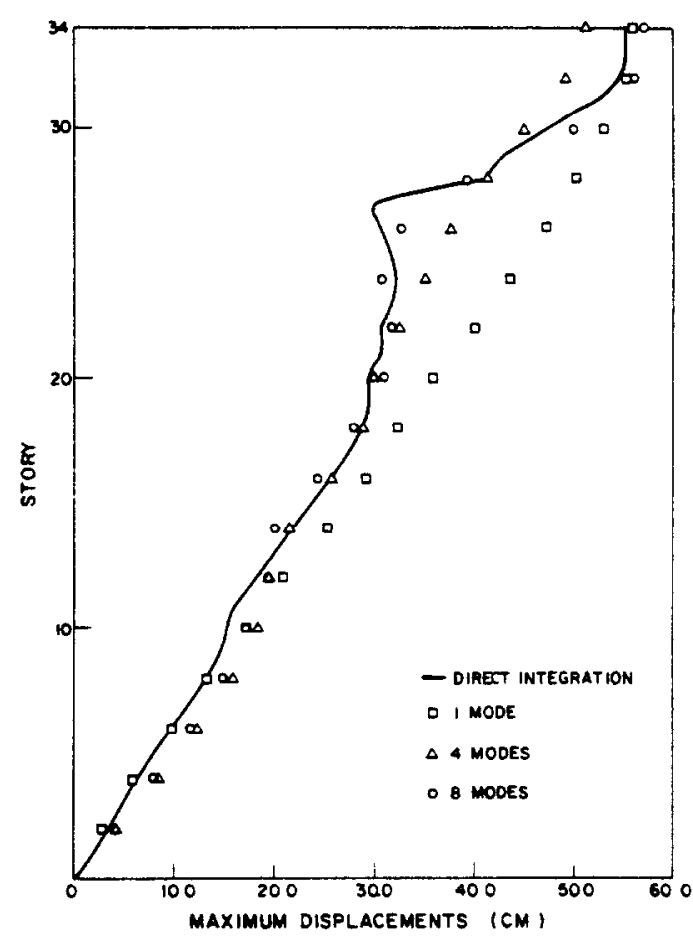

Fig. 8. Thirty-four story building-maximum displacements for each story.

from the $n$ finite element nodal point displacements to the $p$ generalized displacements is effective because $p$ can be much smaller than $n$. In the analysis of a linear system, $p$ would be equal to the number of frequencies $\omega_{i}$ smaller than about 4 times the highest excitation frequency in $R$. We can use this same guideline in the analysis of a nonlinear system, but should recognize that the actual (instantaneous) frequencies of the system are

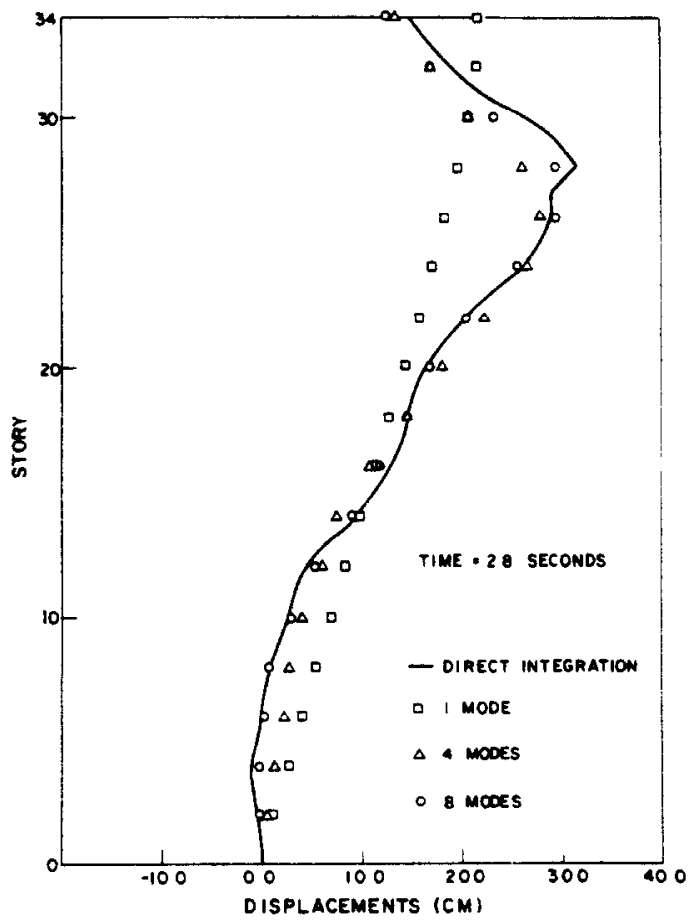

Fig. $9($ a). Thirty-four story building-story displacements at time $=2.8 \mathrm{sec}$.

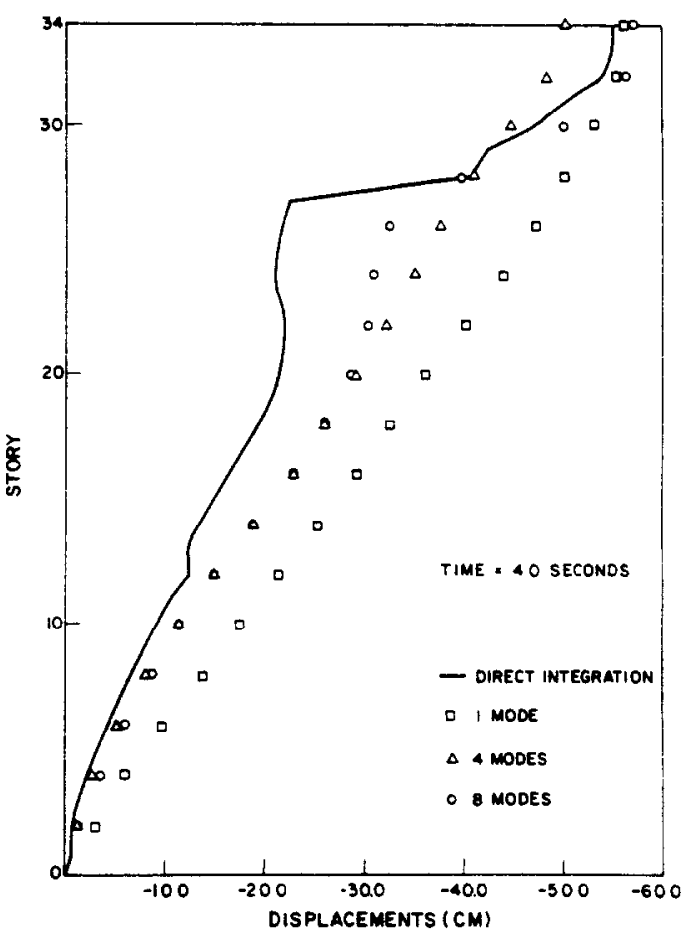

Fig, 9(b). Thirty-four story building-story displacements at time $=4.0 \mathrm{sec}$.

continuously changing during the nonlinear response. If the system stiffens during the response history, the frequencies become larger and the number $p$ selected based on a linear analysis will probably be conservative. However, in the analysis of a softening structure (elas-

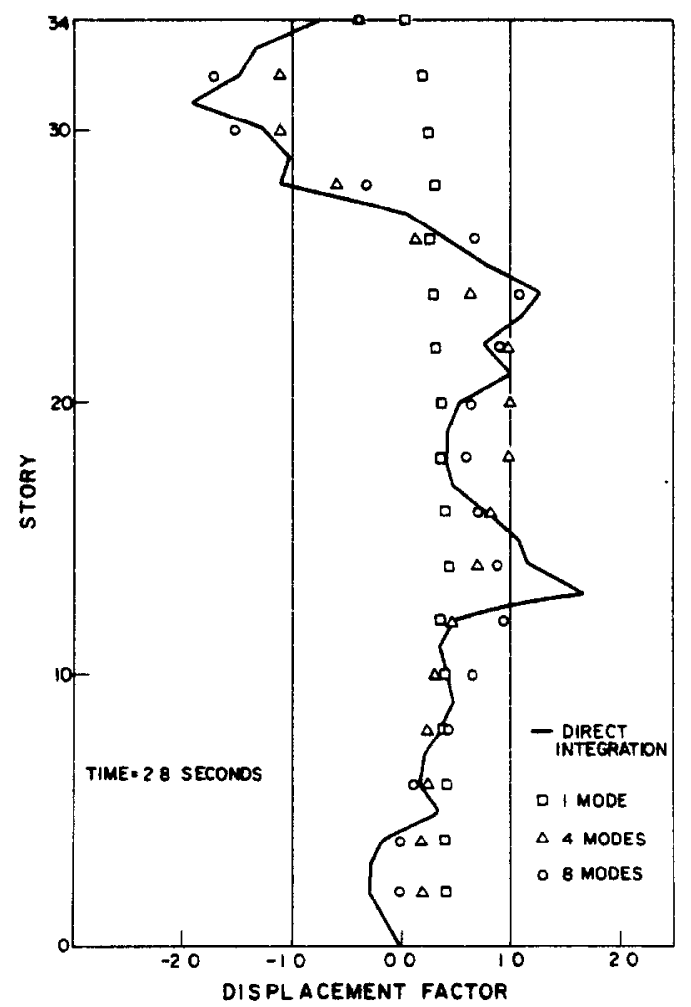

Fig. 10(a). Thirty-four story building-plot of displacement factor for each story at time $=2.8 \mathrm{sec}$ (displacement factor $=$ strain/initial yield strain). 


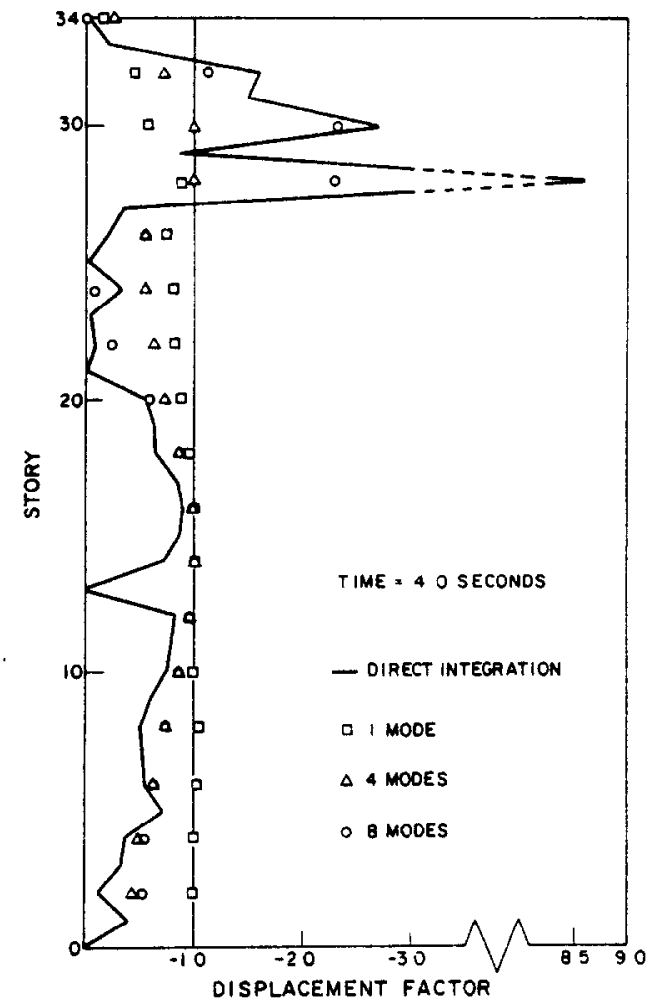

Fig. 1O(b). Thirty-four story building-plot of displacement factor for each story at time $=4.0 \mathrm{sec}$. (displacement factor $=$ strain/initial yield strain).

tic-plastic conditions), the number of generalized displacements may have to be considerably larger than in linear analysis.

Although we call the analysis procedure "mode superposition" it is appropriate to look at the method simply as a coordinate transformation. Hence, the number of transformation vectors to be employed must depend on the response expected in the analysis, and the accuracy of the predicted response depends entirely on the "quality" of the transformation vectors used. For this reason, the procedure is probably best employed in the analysis of only slightly nonlinear systems or systems with only local nonlinearities. In this case, the criteria that render a linear analysis by mode superposition effective are still largely applicable to the nonlinear response calculations.

We may also note that the mode superposition procedure may be effective in preliminary analyses of highly nonlinear systems provided these analyses allow somewhat less accuracy than a final response solution.

To assess the actual accuracy attained in a mode superposition analysis we may evaluate an error norm. similar to that one used in linear analysis,

$$
{ }^{t+\Delta t} \varepsilon_{\mathrm{p}}=\frac{\left\|{ }^{t+\Delta t} \mathbf{R}-{ }^{t+\Delta t} \mathbf{F}^{(t)}-\mathbf{M}^{t+\Delta t} \ddot{\mathbf{U}}^{(t)}\right\|_{2}}{\|\mathbf{R}\|_{2}^{(\max )}}
$$

where $(l)$ denotes the last calculated value, $\|a\|_{2}$ denotes the Euclidean norm of $\mathbf{a}$, and $\|{ }^{r} \mathbf{R}_{2}{ }^{\text {(max) }}$ is the maximum value reached over all discrete times $\tau$. (The relation in eqn (27) assumes that the system is subjected to external loads; otherwise, the denominator must be modified.) If $\varepsilon_{p}$ is unacceptably large, the number of mode shapes should be increased.

In another approach a static correction much as in linear analysis may be applied, but the effectiveness of that correction is very problem-dependent [3].

3.2.2 Substructuring. The effectiveness of the substructuring procedure can be assessed by comparing the number of operations required in the solution of eqns (11) and (23). Considering these equations we find that the method is only effective when only local nonlinearities are considered. Let us assume the following system parameters: $n, m=$ number of equations and their halfbandwidth in eqn (11), and $n_{r}, m_{r}=$ number of equations and their half-bandwidth in eqn (23), $n_{r} \ll n$. To compare the number of operations performed in the solution of eqns (11) and (23) in an approximate manner, we recognize that the updating for the nonlinearities is the same in both equations, and we assume that the operations in vector multiplications can be neglected. In this case, an approximate operation count yields the following information:

\section{lumped mass matrix}

eqn (11): $4 \mathrm{~nm}$ operations per equilibrium iteration eqn (23): $\left(2 n_{r} m_{r}+2 n m_{r}\right)$ operations per first iteration $4 n_{r} m_{r}$ operations per subsequent iteration.

\section{consistent mass matrix}

eqn (11): $6 \mathrm{~nm}$ operations per equilibrium iteration eqn (23): $\left(2 n_{r} m_{r}+4 n m_{r}\right)$ operations per first iteration $4 n_{r} m_{r}$ operations per subsequent iteration.

The above operation count does not include the initial operations required to form $\hat{\mathbf{K}}_{r}$ and the additional peripheral processing required in the substructuring solution.

When considering the above information, it is important to note that $m$, may be significantly larger than $m$. It is for these reasons that for the substructuring algorithm to be effective we should have that $n_{r} m_{r} \ll n m$. The above information also shows that the substructuring algorithm is more likely to be effective when a lumped mass matrix is employed in the analysis.

\section{CONCLUDING REMARKS}

The objective in this paper was to discuss the mode superposition and substructuring procedures available in ADINA for analysis of nonlinear dynamic response. We have presented the theory used, the results of some sample solutions and some recommendations on the use of these analysis procedures. Although we have not employed the methdos as yet in the actual solution of large dynamic problems, our discussion in the paper shows that the procedures can be very effective. We, therefore, look forward to hear about the experiences that users of ADINA might make with the procedures in the solution of actual practical problems.

Acknowledgements - We gratefully acknowledge the financial support of the ADINA users group.

\section{REFERENCES}

1. K. J. Bathe. Finite element formulation, modeling and solution of nonlinear dynamic problems. Chapter in Numerical Methods for Pantial Differential Equations, (Edited by S. V. Parter). Academic Press, New York (1979). 
2. K. J. Bathe, S. Bolourchi, S. Ramaswamy and M. D. Snyder, Some computational capabilities for nonlinear finite element analysis. J. Nucl. Engng Design 46, 429-455 (1978).

3. K. J. Bathe, Finite Element Procedures in Engineering Analysis. Prentice Hall, Englewood Cliffs, New Jersey 1981.

4. R. E. Nickell, Nonlinear dynamics by mode superposition. J. Comp. Meth. Appl. Mech. Engng 7, 107-129 (1976).

5. A. K. Noor, Recent advances in reduction methods for nonlinear problems. J. Comput. Structures 13, 31-44 (1980).

6. R. W. Clough and E. L. Wilson, Dynamic analysis of large structural systems with local nonlinearities. J. Comput. Meth. Appl. Mech. Engng 17/18, 107-129 (1979).

7. K. J. Bathe and A. P. Cimento, Some practical procedures for the solution of nonlinear finite element equations. J. Comput. Meth. Appl. Mech. Engng 22, 59-85 (1980).

8. F. E. Peterson and K. J. Bathe, Nonlinear dynamic analysis of reactor core components. Engineering Analysis Corp., Rep. No. S-104.3, Berkeley, California (1972).

9. P. Lukkunaprasit, S. Widartawan and P. Karasudhi, Dynamic response of an elastic-viscoplastic system in modal coordinates. Earthquake Engng Struct. Dyn. 8, 237-250 (1980).

\section{APPENDIX A}

The Newmark Algorithm

The basic assumptions in the Newmark time integration scheme are

$$
\begin{aligned}
& { }^{t+\Delta t} \dot{\mathrm{U}}={ }^{t} \dot{\mathrm{U}}+\left[(1-\delta)^{t} \ddot{\mathrm{U}}+\delta^{t+\Delta t} \ddot{\mathrm{U}}\right] \Delta t \\
& { }^{t+\Delta t} \mathrm{U}={ }^{t} \dot{\mathrm{U}}+{ }^{t} \mathrm{U} \Delta t+\left[\left(\frac{1}{2}-\alpha\right) \cdot{ }^{\prime} \ddot{\mathrm{U}}+\alpha^{t+\Delta t} \ddot{\mathrm{U}}\right](\Delta t)^{2} .
\end{aligned}
$$

The following constants are used in ADINA with the time integration algorithm

$$
\begin{aligned}
& a_{0}=\frac{1}{\left(\alpha \Delta t^{2}\right)} \quad a_{1}=\frac{\delta}{(\alpha \Delta t)} \quad a_{2}=\frac{1}{(\alpha \Delta t)} \quad a_{3}=\frac{1}{(2 \alpha)}-1 \\
& a_{4}=\delta / \alpha-1 \quad a_{5}=\Delta t(\delta / \alpha-2) / 2 \quad a_{6}=a_{0} a_{7}=-a_{2} \\
& a_{8}=-a_{3} \quad a_{9}=\Delta t(1-\delta) \quad a_{10}=\delta \Delta t .
\end{aligned}
$$

Once the solution for ${ }^{t+\Delta t} \mathrm{U}$ has been obtained the corresponding accelerations and velocities are calculated using

$$
\begin{aligned}
& \left.{ }^{t+\Delta t} \ddot{\mathrm{U}}=a_{6}{ }^{t+\Delta t} \mathrm{U}-{ }^{t} \mathrm{U}\right)+a_{7}{ }^{\prime} \dot{\mathrm{U}}+a_{8}{ }^{\prime} \ddot{\mathrm{U}} \\
& { }^{t+\Delta t} \dot{\mathrm{U}}={ }^{\prime} \dot{\mathrm{U}}+a_{9}{ }^{t} \ddot{\mathrm{U}}+a_{10}{ }^{t+\Delta t} \dot{\mathrm{U}} .
\end{aligned}
$$

